\title{
CURRENT SCENARIO OF THERAPEUTICS FOR EBOLA VIRUS DISEASE
}

\author{
${ }^{1}$ ML Arvinda Swamy, ${ }^{2}$ Madhavan P.N. Nair and ${ }^{1}$ Shailendra K. Saxena \\ ${ }^{1}$ CSIR-Centre for Cellular and Molecular Biology (CCMB), Uppal Road, Hyderabad 500007, India \\ ${ }^{2}$ College of Medicine, Florida International University, Miami 33199 FL, USA
}

\begin{abstract}
Currently various countries in Africa, including Liberia, Sierra Leone, Guinea, Nigeria, are facing disaster due to Ebola Virus Disease (EVD), which is primarily caused by Ebola virus. 2014 outbreak of Ebola associated viral haemorrhagic fever has 55-60\% fatality rate. The incubation period of Ebola is below 21 days; once the appearance of symptoms starts the person will be infective. As there is no specific vaccine, antiviral or drugs for treating Ebola resulting in large number of deaths. Most of the recent outbreaks occurred in remote areas of West Africa. Poverty, lack of awareness, access to health centres, human habitats taking its toll in spreading the disease in large scale. Few nucleotide analogues, protease inhibitors, receptor binding, monoclonal antibodies and anticoagulant therapies are exhibiting promising role in inhibiting the Ebola virus in various (in vitro and in vivo) models.
\end{abstract}

Keywords: Ebola, Ebola Virus Disease, EVD, Filovirus, Therapeutics, EHF, Viral Haemorrhagic Fever1

\section{INTRODUCTION}

Currently various countries in Africa, including Liberia, Sierra Leone, Guinea, Nigeria, are facing disaster due to Ebola Virus Disease (EVD). First Ebola outbreak occurred in Sudan (Nzara) and Congo (Yambuku) in the year 1976, concluding in the villages situated near the Ebola River from which the viral haemorrhagic fever got its name Ebola/Ebola Virus Disease (EVD). EVD belongs to Filoviridae which causes fatal Ebola haemorrhagic fever in humans with fatality rate nearly $90 \%$. Several out breaks of Ebola have been reported from East Africa (Sudan: 1976, 1979, 2004) Central Africa (Democratic Republic of Congo, Uganda \& Gabon: 1976, 1977, 1994, 1997, 2000, 2001, 2004, 2007) and in West Africa (Ivory coast: 1995) since its discovery in 1976. The most recent outbreak started in December 2013 and 2014 in eastern sector of the Republic of Guinea resulted in more than 337 deaths and spreading to republic of Liberia (Gatherer, 2014; Muyembe-Tamfum et al., 2012; Dixon and Schafer, 2014) and other countries.
Ebola belongs to family Filoviridae and other members in this group are Marburgvirus and Cuevavirus. Under Ebola virus genus consists of five diverse species Bundibugyo Ebolavirus (BDBV), Zaire Ebolavirus (EBOV), Reston Ebolavirus (RESTV), Sudan Ebolavirus (SUDV) and Taï Forest Ebolavirus (TAFV). BDBV, EBOV and SUDV have been associated with large EVD outbreaks near the Tropical rain forests of Central and West African distant villages, among these three EBOV causes high mortality rates in humans. RESTV and TAFV were not acquainted to cause illness or fatality in human. RESTV is mostly found in Philippines and China (Leroy et al., 2004; 2005).

\section{TRANSMISSION}

Transmission of virus from the wild animals like primates (Chimpanzees, Gorillas and African green monkeys) and fruit bats (Hypsignathus monstrosus, Epomops franqueti, Myonycteris torquata and Pteropodidae) (Groseth et al., 2007) are the natural hosts for the EVD (Fig. 1).

Corresponding Author: Shailendra K. Saxena, CSIR-Centre for Cellular and Molecular Biology (CCMB), Uppal Road, Hyderabad 500007, India 


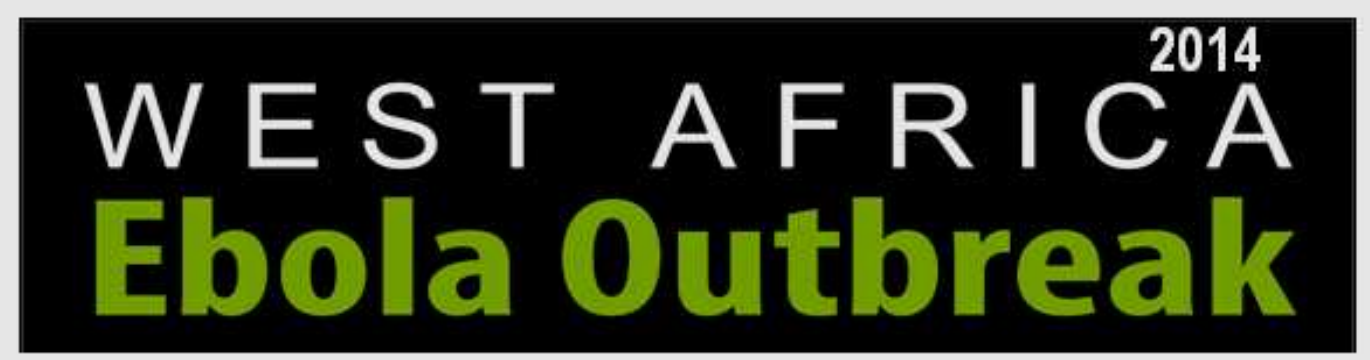

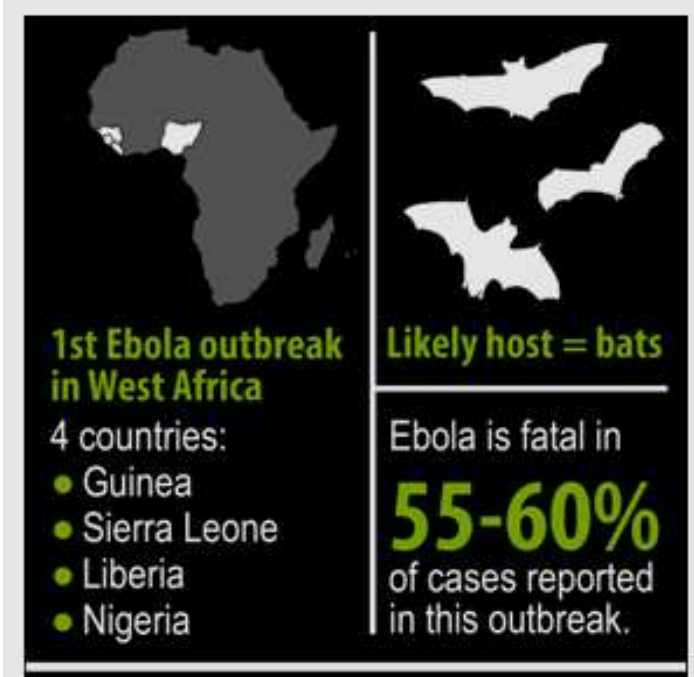

How do you get the Ebola virus?

Direct contact with:

(1) Bodily fluids of a person who is sick with or has died from Ebola. (blood, vomit, pee, poop, sweat, semen, spit, other fluids)

(2) Objects contaminated with the virus (needles, medical equipment)

(3) Infected animals (by contact with blood or fluids or infected meat)

\section{Early Symptoms:}

Ebola can only be spread to others after symptoms begin. Symptoms can appear from 2 to 21 days after exposure.

- Fever

- Headache

- Diarrhea

- Vomiting

- Weakness

\section{When is someone able to spread the disease to others?}

\section{Ebola only spreads} when people are sick. A patient must have symptoms to spread the disease to others.
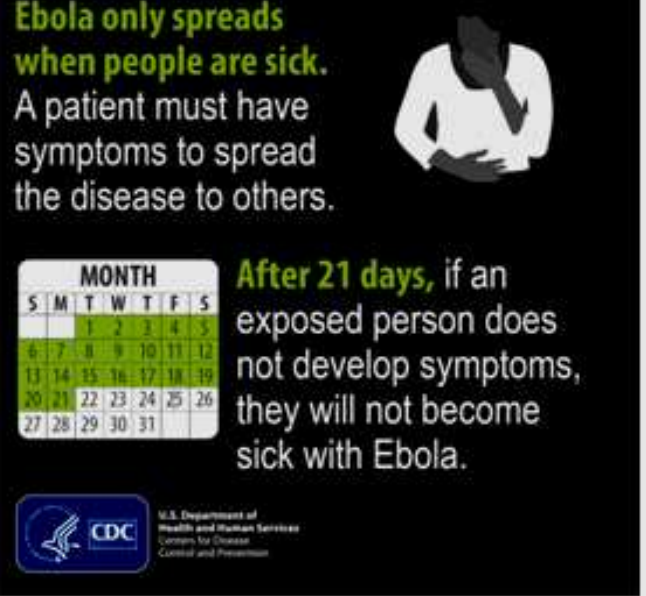

Fig. 1. Infographic exhibiting a basic understanding of the facts behind the Ebola virus including its geographic distribution, (which includes the African countries of Guinea, northern Liberia, Sierra Leone and Nigeria), the virus's likely host, (which seems to be bats), the mortality rate of Ebola Hemorrhagic Fever (EHF), how one becomes infected by the virus, the early symptoms of EHF and when it is possible for a patient ill with EHF to spread the virus to another human being. EHF is one of numerous viral hemorrhagic fevers. It is a severe, often fatal disease in humans and nonhuman primates (such as monkeys, gorillas and chimpanzees). EHF is caused by infection with a virus of the family Filoviridae, genus Ebolavirus. When infection occurs, symptoms usually begin abruptly. The first Ebolavirus species was discovered in 1976 in what is now the Democratic Republic of the Congo near the Ebola River. Since then, outbreaks have appeared sporadically. (Courtesy of Centers for Disease Control and Prevention (CDC), USA, ID\#17677) 
Transmission from primates to humans occurs through the activities like handling ill/infected, butchering, eating, contact through blood from these animals and subsequently viral infection spreads from humans via human to human transmission (Leroy et al., 2004; Groseth et al., 2007).

\section{GENOME OF EBOLA}

Ebola virus is $970 \mathrm{~nm}$ long and filamentous in nature, its diameter is around $80 \mathrm{~nm}$ with (-) ve stranded RNA. Genome of EVD is nearly $19 \mathrm{~kb}$ in size which encodes for seven proteins NP (Nucleoprotien) vp35, vp40, GP (Glycoprotein), vp30, vp24 and L (Polymerase).

How Ebola virus attacks the human system is not completely elucidated. Ebola virus attaches to the host receptors through the GP (glycoprotein) mediated endocytosis into the host vesicles, fusion with the membrane leads to the release of Ribonucleo-capsid into the cytoplasm. Viral RNA dependent RNA polymerase binds to the leader region of the genome to start the sequential transcription of genes. The gene products (mRNA) are protected from the host proteins by capping and polyadenylation by the $\mathrm{L}$ protein during the synthesis (Stahelin, 2014; Watt et al., 2014). Replication starts only when enough nucleoprotein to encapsidated the newly synthesized genomes budding of the virus occurs with, interaction of matrix protein and nucleocapsid under plasma membrane with the help of host ESCRT complexes (McDonald and Martin-Serrano, 2009; Grove and Marsh, 2011).

\subsection{Signs and Symptoms}

EVD is a severe acute viral illness often characterized by the sudden onset of fever, intense weakness, muscle and joint pain, headache and sore throat. This is followed by vomiting, diarrhoea, rash, impaired kidney and liver function and in some cases, both internal and external bleeding. Laboratory findings include low white blood cell and platelet counts and elevated liver enzymes. Few patients may experience rash, red eyes, hiccups, chest pain, cough, sore throat, difficulty in swallowing, difficulty in breathing un explained internal and external bleeding. Incubation period ranges from 2 to 21 days, people are only infective once the symptomatic phase starts and viral transmission can occur through blood and secretions from the patients (Roddy et al., 2012; Dixon and Schafer, 2014).

\section{TARGETTING VIRAL PROTEINS}

\subsection{Protease Inhibitors}

VP30 is essential for transcription of Ebola and it is phosphorylated at N-terminal serine clusters and threonine residues at positions 143 and 146. Host cellular Protein Phosphatase 1 (PP1) controls VP30 dephosphorylation as expression of a PP1-binding peptide cdNIPP1 increased VP30 phosphorylation. 1E703 compound targeted at non-catalytic site of PP1 inhibited the transcription and replication in cell culture model (Ilinykh et al., 2014). Phosphorodiamidate Morpholino Oligomers (PMOs) are short nucleotides binds to the RNA which can alter the RNA-RNA interactions and RNA- Protein interactions which effects viral transcription and translation. The AVI-7537 PMO has been effective in targeting the VP24 gene of Ebola virus (Iversen et al., 2012).

\subsection{Nucleotide Inhibitors}

BCX4430 a nucleoside analogue act as broad spectrum antiviral compound which can be a potential candidate in treatment of patient in delayed/later stages of infection (Warren et al., 2014).

\subsection{Biologically Active Compounds/Antivirals}

Fullerene sugar balls (anti adhesive agents) are the new class of biologically active compounds derived from C60 core they bind to the lectins through glycoside cluster effect which are effective against bacteria. Mannosylated fullerene sugar balls can be used as antivirals as they have shown effect on Ebola pseudotyped infection model. (Nierengarten and Nierengarten, 2014). Griffithsin (lectin) derived from red alga binds to the N-linked Glycans found on the surface of viruses like HIV, HCV, SARS and Ebola virus can be used as antiviral after proper validation (Barton et al., 2014). Favipiravir (T-705) a pyrazinecarboxamide derivateive inhibited the replication of Ebola in later stages of infection thereby decreasing the pathogenesis and disease severity and it has effective against E718 strain (Aerosol Ebola virus) in both cell culture and animal model. This is broad spectrum antiviral administered through oral route it is effective against influenza and it is under phase III clinical trials for the same (Smither et al., 2014; Oestereich et al., 2014). Kinase inhibitors (Genistein and Tryphostin) AG1478 pre-treated host cells with this inhibited the infection of Ebola virus which can be used as broad spectrum antiviral (Kolokoltsov et al., 2012). 


\subsection{Targetting Host Cellular Proteins}

Endoplasmic reticulum $\alpha$-glucosidases I and II are essential for viral maturation and release of virion. The glycolysated viral envelope proteins uses calnexin mediated folding pathway and the compound CM-10-18 (imino sugar) and its derivatives are $\alpha$-glucosidase inhibitors which have shown effect against various hemorrhagic fevers can be used as potent antiviral (Chang et al., 2013). Clomiphene and cationic amphiphiles (Ro 48-8071, Terconazole, Triparanol, AY 9944, Amorolfine and U18666A) induces defects in Niemann-Pick C1 Protein (NPC1) required for viral entry but they have to use in higher concentrations and various combinations of cationic amphiplhies to block the viral entry (Shoemaker et al., 2013). Nedd4 E3 ubiquitin ligase of host protien was utilized by viral PPXY late (L) domain of filoviruses and other RNA viruses like arena virus and rhabdo viruses in budding. $\mathrm{L}$ domain of PPxY can be of ideal choice for blocking the budding and filoviruses infections (Han et al., 2014). Studies on macrophages and dendritic cells with pre-treatment of pyridinyl imidazole p38 MAPK inhibitors can block the entry and infection of EBOV (Johnson et al., 2014).

\subsection{Antibody/Monoclonal Antibodies Against Ebola}

Recombinant adenovirus expressing the Glycoprotein (GP) of Ebola virus has elicited good humoral/antibody response against Ebola virus in wild type and interferon $\alpha / \beta$ receptor knock-out mouse (O'Brien et al., 2014). $\mathrm{ZMAb}$, combination of three EBOV-GP-specific monoclonal antibodies were administered $24-48 \mathrm{~h}$ post exposure to Ebola virus in primates were survived after re challenged them with virus at 10 weeks and 13 weeks are also survived (Qiu et al., 2013).

\section{FUTURE DIRECTIONS}

Recent outbreak of Ebola was the outcome of complex human behavioral, environmental factors on the public. There are many drugs which are under clinical and at pre-clinical level can target both viral and human host proteins. Development of rapid diagnostic tools should be given priority. Establishment of a system for continuous surveillance of emerging and re-emerging diseases is a need of the hour. The cost effective drugs should have global acceptance, accessibility, affordability. The awareness programmers should be conducted in large scale to eradicate the disease and we need to proceed with sense of urgency.

\section{ACKNOWLEDGMENT}

Researchers are grateful to Director, CCMB and Council of Scientific and Industrial Research (CSIR), India for the encouragement and support for this work. SK Saxena is also supported by NIH Awards (R37DA025576; R01MH085259).

\section{REFERENCES}

Barton, C., J.C. Kouokam, A.B. Lasnik, O. Foreman and A. Cambon et al., 2014. Activity of and effect of subcutaneous treatment with the broad-spectrum antiviral lectin griffithsin in two laboratory rodent models. Antimicrob Agents Chemother, 58: 120127. DOI: $10.1128 / A A C .01407-13$

Chang J., T.K. Warren, X. Zhao, T. Gilla and F. Guo et al., 2013. Small molecule inhibitors of ER $\alpha$ glucosidases are active against multiple hemorrhagic fever viruses. Antiviral Res., 98: 432-440. DOI: 10.1016/j.antiviral.2013.03.023

Dixon, M.G. and I.J. Schafer, 2014. Centers for Disease Control and Prevention (CDC). Ebola viral disease outbreak-West Africa, 2014. MMWR Morb Mortal Wkly Rep., 63: 548-551.

Dixon, M.G. and I.J. Schafer, 2014. Centers for Disease Control and Prevention (CDC). Ebola viral disease outbreak-West Africa, 2014. MMWR Morb Mortal Wkly Rep., 63: 548-51.

Gatherer, D., 2014. The 2014 Ebola virus disease outbreak in West Africa. J. Gen. Virol., 95: 16191624. DOI: 10.1099/vir.0.067199-0

Groseth, A., H. Feldmann and J.E. Strong, 2007. The ecology of Ebola virus. Trends Microbiol., 15: 408416. DOI: 10.1016/j.tim.2007.08.001

Grove, J. and M. Marsh, 2011. The cell biology of receptor-mediated virus entry. J. Cell Biol., 195: 1071-1082. DOI: 10.1083/jcb.201108131

Han, Z., J. Lu, Y. Liu, B. Davisb and M.S. Lee et al., 2014. Small-molecule probes targeting the viral PPxY-Host Nedd4 interface block egress of a broad range of RNA viruses. J. Virol., 88: 7294-7306. DOI: $10.1128 /$ JVI.00591-14

Ilinykh, P.A., B. Tigabu and A. Ivanov, 2014. Role of protein phosphatase 1 in dephosphorylation of Ebola virus VP30 and its targeting for the inhibition of viral transcription. J. Biol. Chem., 289: 22723 22738. DOI: $10.1074 /$ jbc.M114.575050

Iversen, P.L., T.K. Warren, J.B. Wells, N.L. Garza and D.V. Mourich et al., 2012. Discovery and early development of AVI-7537 and AVI-7288 for the treatment of Ebola virus and Marburg virus infections. Viruses, 4: 2806-2830. DOI: 10.3390/v4112806 
Johnson, J.C., O. Martinez, A.N. Honko, L.E. Hensley and G.G. Olinger et al., 2014. Pyridinyl imidazole inhibitors of p38 MAP kinase impair viral entry and reduce cytokine induction by Zaire Ebola virus in human dendritic cells. Antiviral Res., 107: 102-109. DOI: 10.1016/j.antiviral.2014.04.014

Kolokoltsov, A.A., S. Adhikary, J. Garver, L. Johnson and R.A. Davey et al., 2012. Inhibition of Lassa virus and Ebola virus infection in host cells treated with the Kinase inhibitors Genistein and tyrphostin. Arch Virol., 157: 121-127. DOI: 10.1007/s00705011-1115-8

Leroy, E.M., B. Kumulungui and X. Pourrut, 2005. Fruit bats as reservoirs of Ebola virus. Nature, 438: 575-576.

Leroy, E.M., P. Rouquet and P. Formenty, 2004. Multiple Ebola virus transmission events and rapid decline of central African wildlife. Science. 303(5656): 387-390. DOI: 10.1126/science.1092528

McDonald, B. and J. Martin-Serrano, 2009. No strings attached: The ESCRT machinery in viral budding and cytokinesis. J. Cell Sci., 122: 2167-7217. DOI: $10.1242 /$ jcs.028308

Muyembe-Tamfum, J.J., S. Mulangu, J. Masumu, J.M. Kayembe and A. Kemp et al., 2012. Ebola virus outbreaks in Africa: Past and present. Onderstepoort. J. Vet. Res., 79: 1-8. DOI: 10.4102/ojvr.v79i2.451

Nierengarten, I. and J.F. Nierengarten, 2014. Fullerene sugar balls: a new class of biologically active fullerene derivatives. Chem. Asian J., 9: 1436-1444. DOI: 10.1002/asia.201400133

O'Brien, L.M., M.G. Stokes, S.G. Lonsdale, D.R. Maslowski and S.J. Smither et al., 2014. Vaccination with recombinant adenoviruses expressing Ebola virus glycoprotein elicits protection in the interferon alpha/beta receptor knock-out mouse. Virology, 452-453: 324-333. DOI: 10.1016/j.virol.2013.03.028

Oestereich, L., A. Lüdtke, S. Wurr, T. Rieger and C.M. Fontela et al., 2014. Successful treatment of advanced Ebola virus infection with T-705 (favipiravir) in a small animal model. Antiviral Res., 105: 17-21. DOI: 10.1016/j.antiviral.2014.02.014
Roddy, P., N. Howard, M.D. Van Kerkhove, J. Lutwama and J. Wamala et al., 2012. Clinical Manifestations and case management of ebola haemorrhagic fever caused by a newly identified virus strain, Bundibugyo, Uganda, 2007-2008. PLoS One, 7: e52986. DOI: 10.1371/journal.pone.0052986

Shoemaker, C.J., K.L. Schornberg, S.E. Delos, C. Scully and H. Pajouhesh et al., 2013. Multiple cationic amphiphiles induce a Niemann-Pick C phenotype and inhibit Ebola virus entry and infection. PLoS One., 8: e562658. DOI: 10.1371/journal.pone.0056265

Smither, S.J., L.S. Eastaugh, J.A. Steward, M. Nelson and R.P. Lenk et al., 2014. Post-exposure efficacy of Oral T-705 (Favipiravir) against inhalational Ebola virus infection in a mouse model. Antiviral Res., 104: 153-155. DOI: 10.1016/j.antiviral.2014.01.012

Stahelin, R.V., 2014. Membrane binding and bending in Ebola VP40 assembly and egress. Front Microbiol., 5: 300 .

Warren, T.K., J. Wells, R.G. Panchal, K.S. Stuthman and N.L. Garza et al., 2014. Protection against filovirus diseases by a novel broad-spectrum nucleoside analogue BCX4430. Nature, 508: 402-405. DOI: 10.1038/nature 13027

Watt, A., F. Moukambi, L. Banadyga, Allison Groseth and Julie Callison et al., 2014. A novel life cycle modeling system for Ebola virus shows a genome length-dependent role of VP24 in virus infectivity. J. Virol., 88: 10511-10524. DOI: 10.1128/JVI.01272-14

Qiu, X., J. Audet, G. Wong, L. Fernando and A. Bello et al., 2013. Sustained protection against Ebola virus infection following treatment of infected nonhuman primates with ZMAb. Sci. Rep., 3: 3365. DOI: 10.1038/srep03365 